\title{
Band-filling and disorder effects in the ordered phase of the Falicov-Kimball model
}

\author{
R. D. B. Carvalho and M. A. Gusmão \\ Instituto de Física, Universidade Federal do Rio Grande do Sul, C.P. 15051, 91501-970 Porto Alegre, Brazil
}

(Received 5 June 2014; revised manuscript received 21 July 2014; published 11 August 2014)

\begin{abstract}
We investigate the stability of "magnetic" ordering against band-filling changes and Anderson-like disorder in the Falicov-Kimball model, within dynamical mean-field theory (DMFT). The one-particle density of states is obtained by both arithmetic and geometric averages over disorder, allowing us to detect the localization transition. Varying the Coulomb interaction and disorder strength, we construct phase diagrams where we identify metallic and insulating regions, with or without magnetic ordering, and determine how these phases are affected by band filling.
\end{abstract}

DOI: 10.1103/PhysRevB.90.085108

PACS number(s): 71.10.Fd, 71.23.-k, 71.30.+h, 75.10.-b

\section{INTRODUCTION}

Disorder effects on strongly correlated electron systems have received much attention for many years [1-3]. The problem is of great relevance for many real materials, like high-temperature oxide supercondutors, since doping, which is necessary to change the band filling, naturally introduces some amount of disorder in the crystal structure. This disorder can be viewed as locally affecting one-electron energies in tight-binding models. In the noninteracting limit, this was the subject of Anderson's pioneering work [4] that led to the concept of Anderson localization. The resulting insulating state is of a completely different nature with respect to a Mott insulator [5], which is due to correlation effects induced by strong Coulomb interaction. The presence of both mechanisms of metal-insulator transition [6] (MIT) in narrow-band solids has led many researchers to investigate the interplay between disorder and electronic correlations in model systems. The models are essentially based on the one-band Hubbard Hamiltonian [7], including disorder as a distribution of on-site energies, as proposed by Anderson [4].

Among a multitude of theoretical approaches, dynamical mean-field theory (DMFT) [8-10] is already well established as one of the most powerful methods to treat local correlations due to strong on-site Coulomb interaction. Information about disorder effects is obtained by a now standard method associated to DMFT in which one evaluates both arithmetic and geometric averages of the local density of states (DOS) [11]. These two averages give the total single-particle spectrum and the distribution of extended states, respectively.

DMFT provides an exact self-consistent mapping of the lattice problem to an effective single-site (or impurity) problem in the limit of infinite dimensions. However, the latter has no closed analytic solution in general, so that many approximate (mostly numerical) impurity solvers have been employed [12]. The only known truly exact solution of a correlated problem in infinite dimensions [13-17] is for the Falicov-Kimball (FK) model [18], which consists of two kinds of spinless fermions, one mobile and one not, that interact locally via Coulomb repulsion. This is equivalent to a simplified Hubbard model [19] in which electrons in a given spin state (e.g., spin down) are frozen, while the opposite-spin electrons can hop through the lattice. This amounts to suppressing spin-flip processes in the dynamics of moving electrons. The antiferromagnetic (AF) order of the Hubbard model at half-filling appears in the FK model as a chessboard pattern of the frozen fermions, which occupy alternating sites on a bipartite lattice. Then correlation implies that the moving ones become preferably localized at the sites of the other sublattice. Here we will freely refer to this state as magnetic order or AF phase.

It is important to emphasize that we are not studying a generic FK model, in which case various charge orderings or charge segregation would be possible $[20,21]$ for fixed values of the density of nonmoving particles (usually called ions) and varying concentration of the moving particles (electrons). In our case, the only difference between these two kinds of particles is their spin state. Their average numbers will be kept equal in both the paramagnetic and AF states, since any difference would imply a non-zero total spin, and hence ferromagnetism. Our choice to focus on the AF phase is kept consistent by avoiding large values of the Coulomb interaction, for which ferromagnetism would be favored near (but not at) half-filling in the Hubbard model.

Inclusion of Anderson-like on-site energy disorder in the FK model yields the so-called Anderson-Falicov-Kimball model. Its electronic properties and phase diagram have been studied in some detail, mainly for the half-filling case, with [22] or without [3,23] magnetic order, but also for variable filling in the nonmagnetic case [24]. In the present paper, we complement these previous studies, allowing for the presence of an AF phase, whose stability is self-consistently investigated both with respect to temperature and the combined effects of Coulomb interaction, energy disorder, and band filling.

The paper is organized as follows. In Sec. II, we present the model and the DMFT approach to solve it, including its formulation for the AF case, and the disorder averages of the DOS. Section III describes our results for the DOS at various band fillings, typical values of the Coulomb interaction $U$, and a broad range of values of disorder strength $\Delta$. It also includes $\Delta$ versus $U$ phase diagrams for different filling fractions. Final comments and conclusions are presented in Sec. IV.

\section{MODEL AND METHOD}

The Anderson-Falicov-Kimball model can be represented by the Hamiltonian

$$
H=\sum_{i} \varepsilon_{i}\left(n_{i}^{c}+n_{i}^{f}\right)-t \sum_{\langle i j\rangle} c_{i}^{\dagger} c_{j}+U \sum_{i} n_{i}^{c} n_{i}^{f},
$$


where we employ the usual notation of creation, annihilation, and number operators (in the Wannier representation) for two kinds of spinless fermions. The $c$ fermions can move with a nearest-neighbor hopping integral $t$, and experience Coulomb repulsion $U$ by the non-moving $f$ fermions. The local energies $\varepsilon_{i}$ are random, with a uniform probability distribution of width $\Delta$.

The exact solution of the Falicov-Kimball model in infinite dimensions $[13,16,17]$ is an example of DMFT with an analytic solution of the impurity problem. It yields a closed form for the $c$-particle Green's function at a finite temperature $T$,

$$
G_{i i}\left(i \omega_{n}\right)=\frac{1-p}{i \omega_{n}-\bar{\varepsilon}_{i}-\lambda\left(i \omega_{n}\right)}+\frac{p}{i \omega_{n}-\bar{\varepsilon}_{i}-U-\lambda\left(i \omega_{n}\right)},
$$

where $\omega_{n}=(2 n+1) \pi T$, for integer $n$, are Matusbara frequencies, $\bar{\varepsilon}_{i} \equiv \varepsilon_{i}-\mu$ is the local energy measured with respect to the chemical potential, $p$ measures the probability of finding an $f$ particle at site $i$, and $\lambda\left(i \omega_{n}\right)$ are the Fourier components of the dynamical mean-field connecting this site to the $c$-particle reservoir that replaces the lattice. Clearly $p=\left\langle n_{f}\right\rangle$, the average number of $f$ particles per site, and must be determined self-consistently. Keeping in mind the analogy of $c$ and $f$ fermions with spin-up and spin-down electrons in the Hubbard model, the sum $\left\langle n_{c}\right\rangle+\left\langle n_{f}\right\rangle$ must equal the total number of particles per site, the band-filling fraction $n$.

\section{A. Paramagnetic solution}

It is easier to begin with the nonmagnetic case, for which the self-consistent solution implies that $p=n / 2$. Then the only unknown in Eq. (2) is the dynamical mean field $\lambda\left(i \omega_{n}\right)$, which can be determined from the self-consistent condition that the site-diagonal lattice Green's function must be equal to the effective single-site solution with the same self-energy $\Sigma_{i}\left(i \omega_{n}\right)$, that is purely local in the limit $d \rightarrow \infty$. With disorder, this only makes sense if the lattice Green's function is averaged over the distribution of local energies $\varepsilon_{i}$, and we have the self-consistence condition

$$
G_{i i}^{\mathrm{av}}\left(i \omega_{n}\right)=\int \frac{\rho_{0}(\epsilon) d \epsilon}{\left[G_{i i}^{\mathrm{av}}\left(i \omega_{n}\right)\right]^{-1}+\lambda\left(i \omega_{n}\right)-\epsilon}
$$

complemented by the disorder average of Eq. (2). Here, the $i i$ subscript does not imply any site dependence, but just indicates the local (site-diagonal) Green's function.

Since the averaging is not done directly on the Green's function but on the DOS, we perform the analytic continuation $i \omega_{n} \rightarrow \omega+i 0^{+}$to obtain the retarded single-site Green's function, $G_{i i}(\omega)$, which yields the local spectral density $\rho_{i}(\omega)=-\operatorname{Im} G_{i i}(\omega) / \pi$. This is then averaged over disorder to evaluate the spectral density, or local DOS, $\rho_{\mathrm{av}}(\omega)$, from which the disorder-averaged local Green's function is obtained through its spectral representation

$$
G_{i i}^{\mathrm{av}}(\omega)=\int \frac{\rho_{\mathrm{av}}(\epsilon) d \epsilon}{\omega-\epsilon+i 0^{+}} .
$$

In practice, we work only with retarded Green's functions, and the dynamical mean field is also a function $\lambda(\omega)$ of real frequencies.
As we mentioned before, we must use two kinds of averages. Denoting arithmetic average by a line above the sitedependent quantity being averaged, we have $\rho_{a}(\omega)=\overline{\rho_{i}(\omega)}$ and $\rho_{g}(\omega)=\exp \left[\overline{\ln \rho_{i}(\omega)}\right]$ as the arithmetic and geometric averages of the DOS, respectively. Self-consistent determination of the corresponding $G_{a}(\omega)$ or $G_{g}(\omega)$ implies that there exist different hybridization functions $\lambda_{a}(\omega)$ and $\lambda_{g}(\omega)$.

To simplify the numerical calculations, we will consider an uncorrelated DOS corresponding to a Bethe lattice [25] in the limit of infinite coordination number $(z \rightarrow \infty), \rho_{0}(\epsilon)=$ $\frac{4}{\pi W} \sqrt{1-4(\epsilon / W)^{2}}$, where $W=4 t^{*}$ is the band width, with the hopping integral scaled [26] as $t=t^{*} / \sqrt{z}$. Since no hopping loops are possible in the Bethe lattice, the only way for an electron to visit the rest of the lattice is by going out and coming back through the same nearest-neighbor site (any one of them). This implies a simple relation for the hybridization function, $\lambda_{\alpha}(\omega)=z t^{2} G_{\alpha}(\omega)=W^{2} G_{\alpha}(\omega) / 16$, where $\alpha=a, g$. In the following, we will choose the band width as our energy unit, fixing $W=1$.

When self-consistency is achieved for $\lambda(\omega)$, we need to evaluate the average number of $c$ fermions, for which we use the expression

$$
\left\langle n_{c}\right\rangle=\int d \omega \rho_{a}(\omega) f(\omega),
$$

where $f(\omega)$ is the Fermi function. We then adjust the chemical potential and restart the self-consistency cycle until the equality $\left\langle n_{c}\right\rangle=n / 2$ is verified. To fix the chemical potential, one must use arithmetic average since all states (not only the extended ones) contribute to the occupation number.

\section{B. Magnetic order}

We now turn to the AF ordered case, in which the lattice is divided in two sublattices, $A$ and $B$, related by the condition

$$
\left\langle n_{f}\right\rangle_{A}+\left\langle n_{f}\right\rangle_{B}=\left\langle n_{c}\right\rangle_{A}+\left\langle n_{c}\right\rangle_{B}=n .
$$

A convenient order parameter is $M \equiv \frac{1}{2}\left(\left\langle n_{f}\right\rangle_{A}-\left\langle n_{f}\right\rangle_{B}\right)$, which saturates at $n / 2$ in the chessboard ground state. We will refer to this parameter as magnetization.

The local Green's function for $c$ electrons, Eq. (2), keeps its form except for the inclusion of $A$ or $B$ subscripts depending on the sublattice to which the site belongs. The self-consistence condition, Eq. (3), now reads [9]

$$
G_{i i \gamma}^{\mathrm{av}}\left(i \omega_{n}\right)=\frac{\xi}{\xi_{\gamma}} \int \frac{\rho_{0}(\varepsilon)}{\xi-\varepsilon} d \varepsilon,
$$

where $\gamma=A, B$, and

$$
\xi_{\gamma} \equiv\left[G_{i i \gamma}^{\mathrm{av}}\left(i \omega_{n}\right)\right]^{-1}+\lambda_{\gamma}\left(i \omega_{n}\right), \quad \xi \equiv \sqrt{\xi_{A} \xi_{B}} .
$$

In contrast to the homogeneous case, average occupation numbers for both fermion species must be evaluated selfconsistently, as they are no longer fixed at $n / 2$. While $\left\langle n^{c}\right\rangle$ is obtained by Eq. (5), with the appropriate sublattice indices, evaluation of $\left\langle n^{f}\right\rangle$ is more involved since there is no Green's function associated to these particles in the FK model. This would not be the case for the Hubbard model, when both averages could be treated on equal footing, at the price of losing the exact solution. Following early works on the FK 
model [13-17], we write down an expression for $\left\langle n_{i}^{f}\right\rangle$ obtained by the same derivation that yields Eq. (2) from the exact partition function. It can be expressed [27] as a kind of Fermi function,

$$
\left\langle n_{i}^{f}\right\rangle=\frac{1}{e^{\beta\left(E_{i}^{f}-\mu\right)}+1},
$$

where $\beta \equiv 1 / T$ (taking $k_{B}=1$ ), and $E_{i}^{f}$ may be viewed as an effective $f$-fermion energy, defined by

$$
\begin{gathered}
E_{i}^{f} \equiv \varepsilon_{i}+\epsilon_{f}+T \sum_{n}\left[\ln \left(i \omega_{n}-\varepsilon_{i}-\lambda_{n}\right)\right. \\
\left.-\ln \left(i \omega_{n}-\varepsilon_{i}-U-\lambda_{n}\right)\right] e^{i \omega_{n} 0+},
\end{gathered}
$$

where we used the short-hand notation $\lambda_{n} \equiv \lambda\left(i \omega_{n}\right)$. The new energy $\epsilon_{f}$ appearing in Eq. (10) has to be introduced as an adjustable parameter to compensate for the lack of band width (kinetic energy) of $f$ fermions, which in our case are just electrons from the same band but with opposite spin. It is adjusted by imposing the condition (6).

We see now that the global $p=\left\langle n^{f}\right\rangle$ of Eq. (2) appears here as a local quantity, so that it must be averaged over disorder. We do this as a simple (arithmetic) average, since this quantity is not tied to extended states. It has to be done for each sublattice, yielding $p_{A}$ and $p_{B}$.

In order to continue to work with real frequencies, we use a standard procedure to convert Matsubara sums to integrals over the real axis on the complex-frequency plane. The relevant relation is [17]

$$
\begin{aligned}
T & \sum_{n}\left[\ln \left(i \omega_{n}-\epsilon-\lambda_{n}\right)\right] e^{i \omega_{n} 0+} \\
& =-\frac{1}{\pi} \operatorname{Im} \int_{-\infty}^{\infty} d \omega f(\omega) \ln (\omega-\epsilon-\lambda(\omega)),
\end{aligned}
$$

which applies to both terms in Eq. (10), with $\epsilon=\varepsilon_{i}$ or $\epsilon=$ $\varepsilon_{i}+U$.

\section{DOS, MAGNETIZATION, AND PHASE DIAGRAMS}

Having presented the model and the approach to be employed, we begin to discuss our results, obtained via numerical implementation of the outlined algorithm to obtain densities of states and occupations numbers.

We begin with a well-behaved DOS for the half-filled system in the clean limit, at $T=0$, shown in Fig. 1, where we can see the spin polarization characteristic of a magnetic solution. The denominations "up" and "down" are arbitrary. Since this DOS refers to $c$-fermions, and since the down sublattice is occupied (below the Fermi level, $\omega=0$ ), we are viewing the $f$-fermions as spin-up electrons. Notice that the two plots in Fig. 1 correspond to values of the Coulomb interaction $U$ below and above the critical value $U_{c}=0.5$ for the Mott transition in the paramagnetic case. This is seen on the PM DOS (also shown), but no qualitative changes are observable for the AF DOS. It is interesting to notice that for $U<U_{c}$ the insulating state is a consequence of the magnetic order, which changes the periodicity and opens a gap at the Fermi level. In contrast, for $U>U_{c}$ a Mott gap exists even in the PM state, so that one can interpret the magnetism in terms of a usual exchange mechanism for localized spins.

Significant changes occur when one moves away from halffilling and adds disorder. This can be seen in Fig. 2. Even for a large $U$, causing a reasonably large charge gap, the Fermi level falls inside the lower Hubbard sub-band for any $n<$ 1. Thus the system is metallic, but remains magnetic. The effect of disorder is seen, in part, as the expected widening of the arithmetically averaged DOS, which is accompanied by a reduction of the correlation gap. In addition, we also observe a fast shrinking geometrically averaged DOS. On the second plot of Fig. 2 we no longer have extended states at the Fermi level $(\omega=0)$, so that from one plot to the other we observe a transition from a metal to an Anderson-localized insulator.

We want to emphasize that the densities of states shown here are for zero temperature, since we are mainly interested in viewing the effects of disorder and band filling on the groundstate properties. Previous investigations of temperature effects on the DOS at half-filling for the ordered phase [28-30] have shown that metallic behavior might be induced at $T \neq 0$.

The effect of disorder on the magnetism can be better viewed through magnetization versus temperature curves, as shown in Fig. 3 (top panel) for $n=1$. Band-filling effect is exemplified in the bottom panel of the same figure. From these plots it is clear that both doping (departure from half-filling) and disorder tend to suppress the magnetic phase. It is
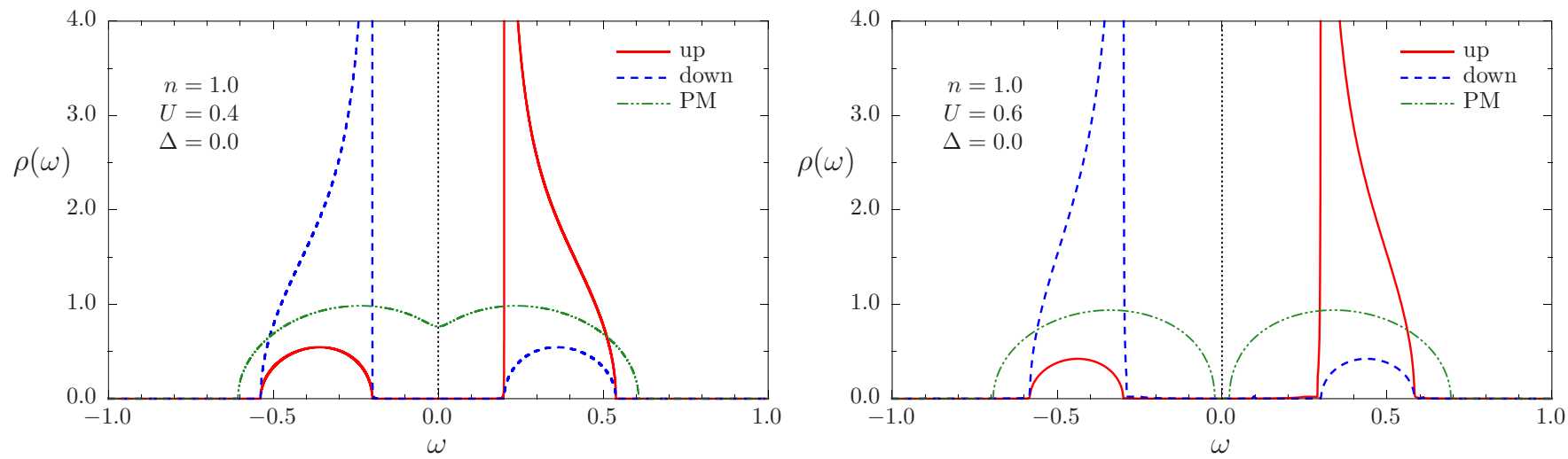

FIG. 1. (Color online) DOS in the magnetic state at half-filling, for two values of the Coulomb interaction $U$, and at $T=0$. The paramagnetic DOS is also plotted for comparison. 

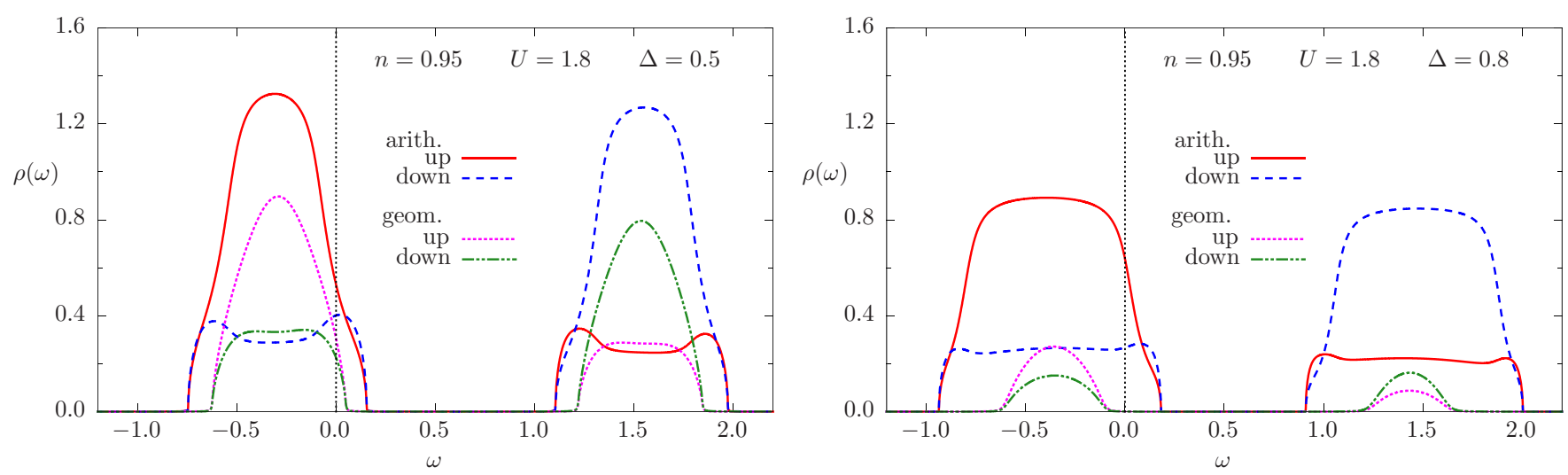

FIG. 2. (Color online) DOS in the magnetic state slightly below half-filling, at $T=0$, for a relatively large $U$ and two values of the disorder strength $\Delta$. We show both arithmetic and geometric averages of the DOS, as well as spin polarization. The Fermi level is indicated by a vertical line at $\omega=0$, which falls inside the first Hubbard subband when $n<1$. A finite value of the geometrically averaged DOS at the Fermi level indicates that the system is still metallic for $\Delta=0.5$, but extended states no longer exist at the Fermi level for $\Delta=0.8$, for which the system is in the Anderson-localization regime and remains magnetic.

worth mentioning that the $M(T)$ curves are not always "well behaved" as those shown in Fig. 3, but anomalous behavior has been described for the half-filled case in the small- $U$ limit $[31,32]$. Our aim here is to show the reduction of the Néel temperature $T_{N}$ with disorder and doping.

On the other hand, one expects the Coulomb interaction to favor a magnetic state. Figure 4 shows the behavior of $T_{N}$
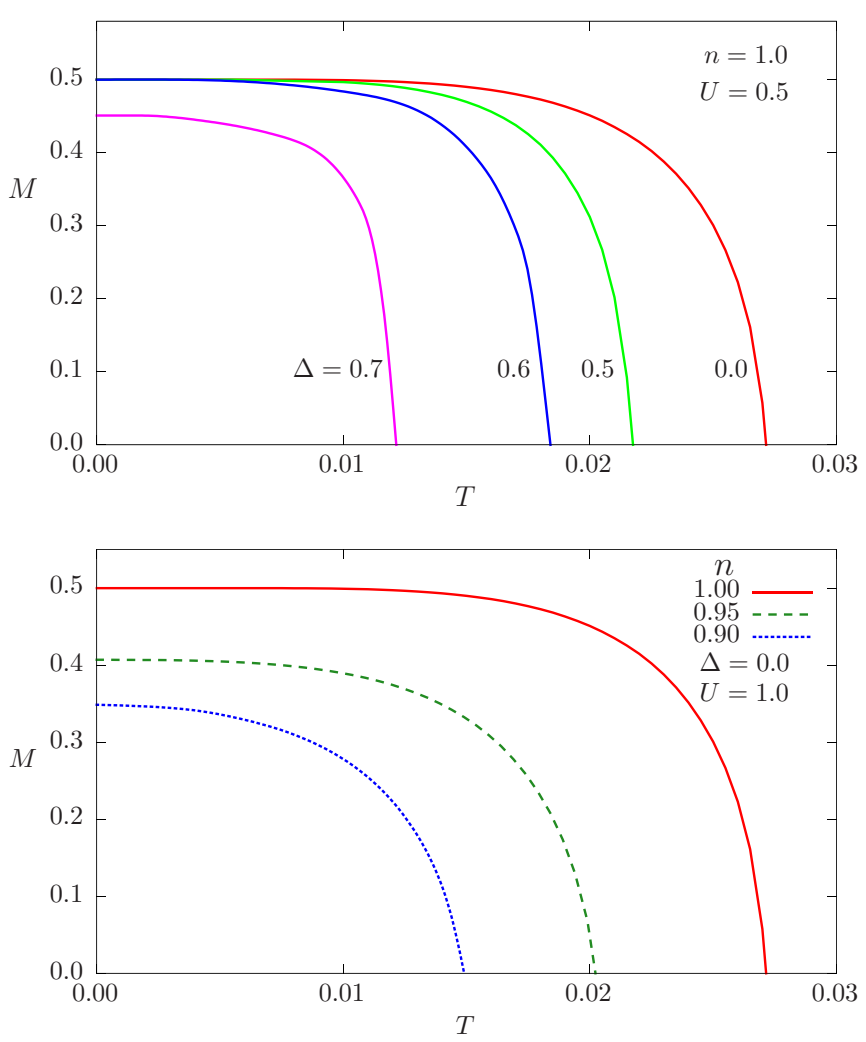

FIG. 3. (Color online) Magnetization as a function of temperature: at half-filling, for different values of $\Delta$ (top) and for three different fillings, in the absence of disorder (bottom). These plots show that $T_{N}$ is reduced by both disorder and doping. as a function of $U$, illustrating combined effects of Coulomb correlations with disorder (top panel) or with doping (bottom panel). At half-filling and no disorder, we can see that the weak-coupling magnetic state observed in Fig. 1 is stable for any nonzero $U$. Both disorder and doping yield finite critical
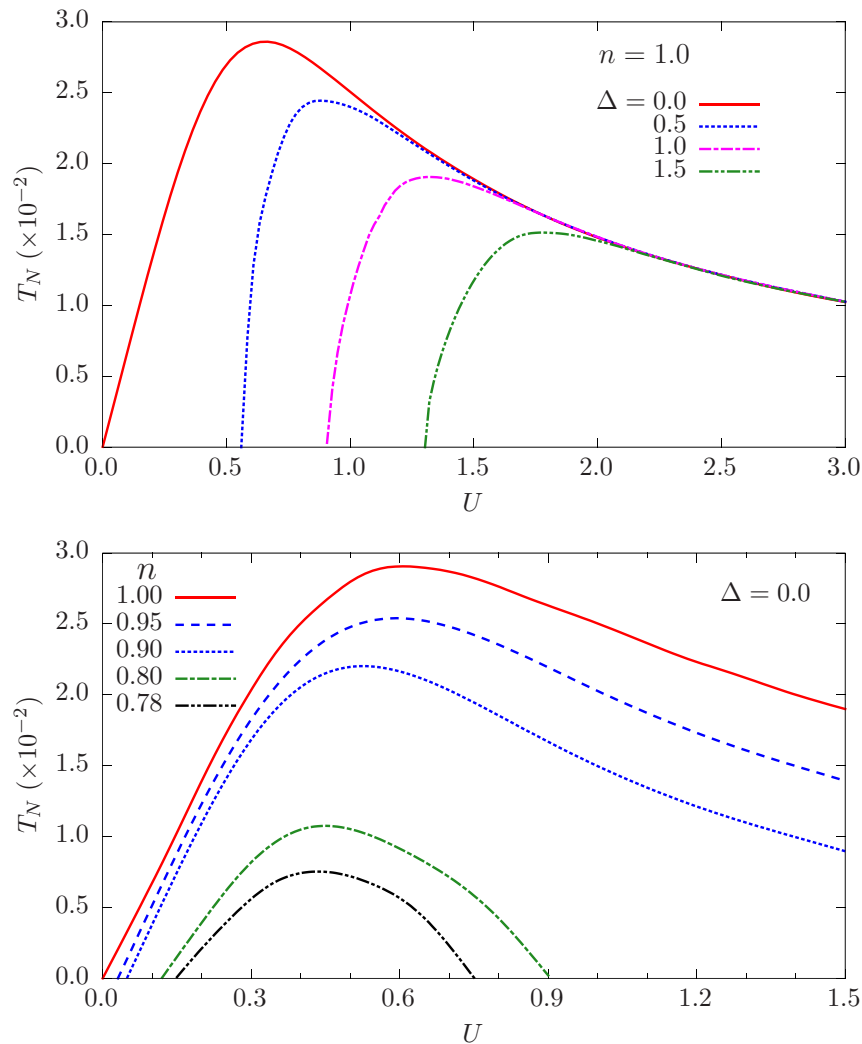

FIG. 4. (Color online) Behavior of the Néel temperature with Coulomb interaction, for different disorder strengths (top) and for different band fillings in the absence of disorder (bottom). We see that magnetic order sets in above a finite critical value of $U$, except at half-filling in the clean limit. For low filling, the magnetic state is also unstable at large $U$. 


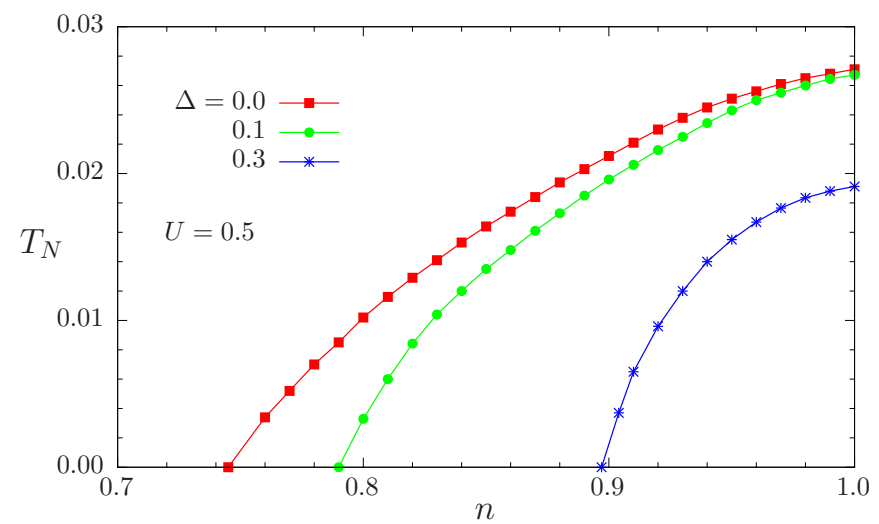

FIG. 5. (Color online) Néel temperature as a function of band filling for some values of the interaction strength $\Delta$, showing that disorder enhances the doping tendency to suppress AF ordering.

values of Coulomb interaction to stabilize a magnetic phase, since the Fermi surface departs from any possible nesting condition that would favor a spin-density-wave state. However, doping also introduces a second critical coupling above which $T_{N} \rightarrow 0$, so that the stability region of AF ordering shrinks fast with doping, as shown in the bottom panel of Fig. 4. The reduction of $T_{N}$ at large $U$ for $n=1$ can be understood as a Heisenberg limit of the Hubbard model (Ising limit in the case of Falicov-Kimball), since the effective exchange interaction between localized electrons in the Mott phase behaves as $J \sim t^{2} / U$. We can then understand why disorder becomes irrelevant for large $U$ at half-filling (top panel of Fig. 4), since we have localized spins whose exchange interaction is not affected by the kind of disorder considered in the model. This regime does not exist away from half-filling, where the system is metallic.

To show the combined effects of disorder and doping, we plot in Fig. 5 the Néel temperature as a function of band filling for some values of $\Delta$. It can be seen that disorder enhances the reduction of $T_{N}$ by doping, contributing to suppress the AF state.

Collecting the information obtained so far, we build up representative zero-temperature phase-diagrams that show the interplay between correlations and disorder in different doping regimes. These phase diagrams are presented in Fig. 6. One can observe that there is a rich variety of phases at half-filling. It is worth mentioning that the lines separating Anderson and metallic regions, or the Anderson and Mott regions at half-filling, are the same that are obtained by imposing a paramagnetic solution [22,24], even though parts of these regions are magnetic here. One of the interesting aspects of the phase diagrams of Fig. 6 is the shape of the metallic region, from which one sees that both correlations and disorder counteract each other's effects to stabilize a metal where either one or the other would favor an insulator.

Another interesting region in Fig. 6 is at the "reentrance" of the Anderson-localization (AL) regime around $\Delta \simeq 1.0, U \simeq$
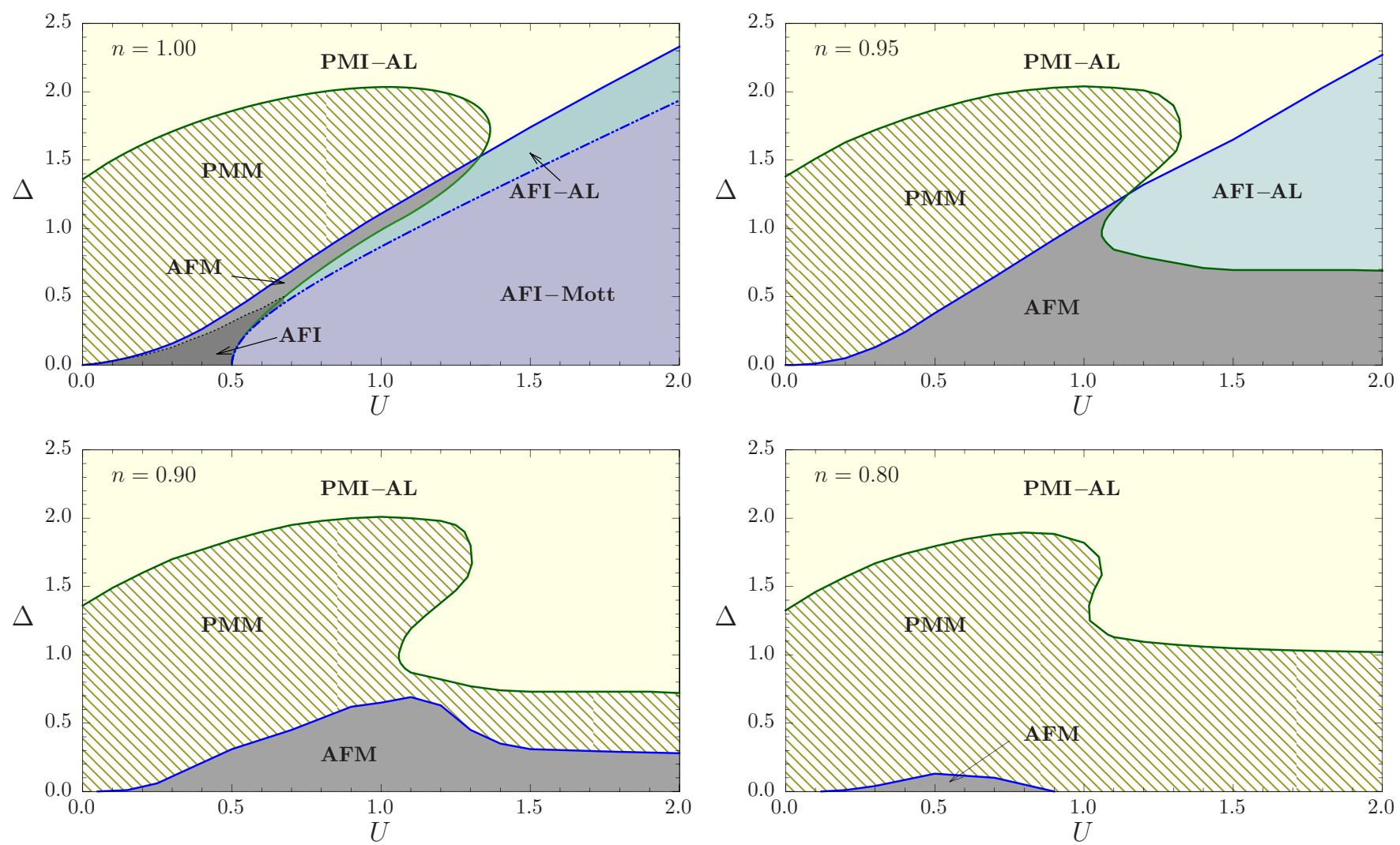

FIG. 6. (Color online) Phase diagrams showing the interplay between disorder and Coulomb interaction for representative band fillings, at $T=0$. We identify the various phases by their magnetic state, PM or AF, followed by a third letter indicating the metallic (M) or insulating (I) nature of the phase, with further identification of Mott and Anderson-localization (AL) regimes. 


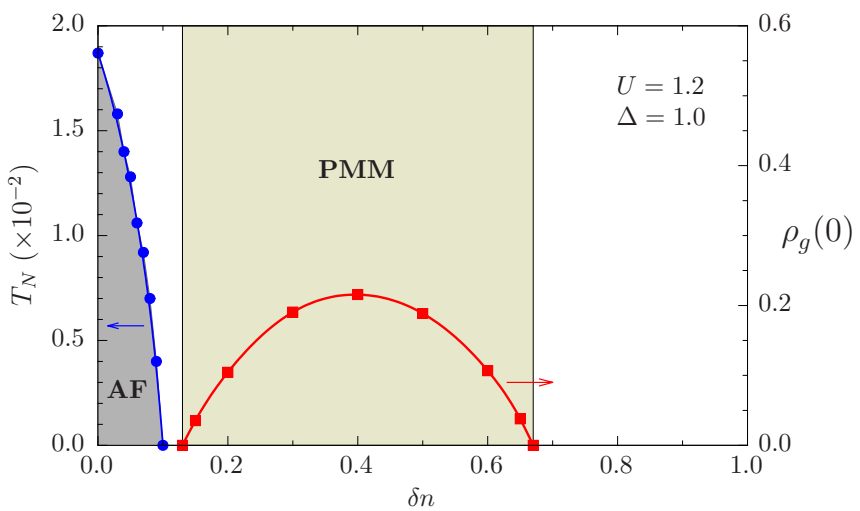

FIG. 7. (Color online) Néel temperature (left $y$ axis) and density of extended states at the Fermi level (right $y$ axis) as functions of doping $(\delta n=1-n)$ for the indicated values of $U$ and $\Delta$. In the white regions the system is a paramagnetic AL insulator.

1.2. At $n=0.95$ this point, which was in the Mott insulating state at $n=1$, corresponds to a disorder-induced insulator with AF magnetic ordering (AFI-AL). At $n=0.90$, the AF solution is no longer stable in that region, and we have a PM Anderson insulator (PMI-AL). When we reach $n=0.80$, the reference point has entered a PM metallic phase (PMM). When further increasing the doping (not shown in Fig. 6) the boundary line between PMI-AL and PMM straightens up and comes down again [24], so that we find the point once more in the PMI-AL state. Its passage through the metallic phase can be monitored by looking at the geometrically averaged DOS at the Fermi level, $\rho_{g}(0)$. This is shown in Fig. 7, where we also show the suppression of magnetic order by plotting the variation of $T_{N}$ with doping. The whole picture looks very similar to famous phase diagrams of high-temperature superconductors and other strongly correlated compounds. However, we want to stress that the relative scales of the two quantities plotted in Fig. 7 are arbitrary, and that the dome-shaped line is not the superconducting critical temperature, but a plot the density of extended states at zero temperature.

\section{CONCLUSIONS}

We have investigated in detail the combined effects of on-site disorder and band filling changes (doping) in the Falicov-Kimball model. Our main focus has been on the chessboard ordered phase of the model. We refer to it as an antiferromagnetic phase in view of the identification of the FK model with a simplified version of the Hubbard model, with the two fermion flavors of the former being associated to the two spin states of conduction electrons in a single correlated band.

Due to the exact solution of the DMFT effective single-site problem in the FK model, we were able to evaluate densities of states directly from real-frequency Green's functions at any temperature. This allowed us to study the stability of the magnetic state against temperature, disorder and doping. In addition to that, the DOS geometrically averaged over disorder, which gives the distribution of extended states, yielded information about the insulating or metallic nature of the states. We could, then, build up phase diagrams in the $U-\Delta$ plane for representative values of band filling. These phase diagrams show a variety of regimes: paramagnetic or antiferromagnetic, metallic or insulating, with the insulating state being induced by correlations or by Anderson localization due to disorder.

Our main observations are that the AF phase is quickly suppressed away from half-filling, this effect being enhanced by increasing disorder. This is better viewed through the variation of the Néel temperature $\left(T_{N}\right)$, which has a maximum at half-filling (zero doping) and falls to zero at a critical doping that depends on the amount of disorder. Moreover, in the filling range where the AF phase is no longer stable (high doping) there is a limited range of electron densities for which a metallic phase exists, characterized by a dome-shaped variation of the density of extended states at the Fermi level as a function of doping. When we combine this information ( $T_{N}$ and DOS) in the same plot (Fig. 7), the result is strongly reminiscent of the phase diagram of oxide superconductors, heavy fermions, and similar materials showing competition between magnetism and superconductivity. Even though we do not investigate here the occurrence of superconductivity, it is obviously inside the metallic phase, indicated by the DOS dome, that a superconducting state can exist. On the other hand, the doping range between these two regimes, which in real systems presents a highly complex scenario, appears here as just an Anderson insulator, which is probably related to the fact that the FK model describes a non-Fermi liquid by construction.

\section{ACKNOWLEDGMENT}

This work was supported in part by Conselho Nacional de Desenvolvimento Científico e Tecnológico (CNPq), Brazil.
[1] P. A. Lee and T. V. Ramakrishnan, Rev. Mod. Phys. 57, 287 (1985).

[2] D. Belitz and T. R. Kirkpatrick, Rev. Mod. Phys. 66, 261 (1994).

[3] K. Byczuk, W. Hofstetter, and D. Vollhardt, Phys. Rev. Lett. 94, 056404 (2005).

[4] P. W. Anderson, Phys. Rev. 124, 41 (1961).

[5] N. F. Mott, Proc. Phys. Soc., London, Sect. A 62, 416 (1949).

[6] M. Imada, A. Fujimori, and Y. Tokura, Rev. Mod. Phys. 70, 1039 (1998).
[7] J. Hubbard, Proc. R. Soc. London, Ser. A 276, 238 (1963).

[8] W. Metzner and D. Vollhardt, Phys. Rev. Lett. 62, 324 (1989).

[9] A. Georges, W. Kotliar, G. Krauth, and M. Rozemberg, Rev. Mod. Phys. 68, 13 (1996).

[10] D. Vollhardt, AIP Conf. Proc. 1297, 339 (2010).

[11] V. Dobrosavljevic, A. A. Pastor, and B. K. Nikolic, Europhys. Lett. 62, 76 (2003).

[12] R. Bulla, Phys. Rev. Lett. 83, 136 (1999).

[13] U. Brandt and C. Mielsch, Z. Phys. B 75, 365 (1989). 
[14] U. Brandt and C. Mielsch, Z. Phys. B 79, 295 (1990).

[15] U. Brandt and C. Mielsch, Z. Phys. B 82, 37 (1991).

[16] V. Janis, Z. Phys. B 83, 227 (1991).

[17] J. K. Freericks and V. Zlatić, Rev. Mod. Phys. 75, 1333 (2003).

[18] L. Falicov and J. Kimball, Phys. Rev. Lett. 22, 997 (1969).

[19] P. G. J. van Dongen and D. Vollhardt, Phys. Rev. Lett. 65, 1663 (1990).

[20] C. Gruber, N. Macris, P. Royer, and J. K. Freericks, Phys. Rev. B 63, 165111 (2001).

[21] J. K. Freericks, Phys. Rev. B 47, 9263 (1993).

[22] M. A. Gusmão, Phys. Rev. B 77, 245116 (2008).

[23] K. Byczuk, Phys. Rev. B 71, 205105 (2005).
[24] R. D. B. Carvalho and M. A. Gusmão, Phys. Rev. B 87, 085122 (2013).

[25] H. Bethe, Proc. R. Soc. London, Ser. A 150, 552 (1935).

[26] E. Müller-Hartmann, Z. Phys. B 76, 211 (1989).

[27] Q. Si, G. Kotliar, and A. Georges, Phys. Rev. B 46, 1261 (1992).

[28] S. R. Hassan and H. R. Krishnamurthy, Phys. Rev. B 76, 205109 (2007).

[29] O. P. Matveev, A. M. Shvaika, and J. K. Freericks, Phys. Rev. B 77, 035102 (2008).

[30] R. Lemański and K. Ziegler, Phys. Rev. B 89, 075104 (2014).

[31] P. G. J. van Dongen, Phys. Rev. B 45, 2267 (1992).

[32] L. Chen, J. K. Freericks, and B. A. Jones, Phys. Rev. B 68, $153102(2003)$. 